\title{
Vedolizumab como terapia de segunda línea para colitis ulcerosa: experiencia de un caso real
}

\section{First line therapy with vedolizumab in ulcerative colitis: Real life experience in one real patient}

\author{
Melanie Figueroa-Palafox e Ylse Gutiérrez-Grobe* \\ Clínica de Enfermedades Digestivas y Obesidad, Fundación Clínica Médica Sur, Ciudad de México, México
}

\section{Resumen}

Se presenta el caso de una paciente de 24 años con colitis ulcerosa de cuatro años de diagnóstico. Inició con hospitalización en 2017, con requerimiento de esteroides intravenosos, con poca respuesta al día 3 de esteroides sistémicos, por lo que se realizó protocolo para manejo con infliximab y se inició terapia biológica, con adecuada respuesta. Sin embargo, a finales del 2019 requiere optimización de frecuencia de dosis por niveles séricos subóptimos, sin presencia de anticuerpos. En octubre del 2020 se observan niveles bajos de infliximab nuevamente, por lo que se decide modificar blanco terapéutico y se inicia vedolizumab como terapia de segunda línea, con remisión clínica y calprotectina fecal negativa a los ocho meses del inicio de la terapia con vedolizumab.

Palabras clave: Colitis ulcerosa. vedolizumab. Terapia de segunda línea.

\begin{abstract}
We present a case of a 24-year-old female, with ulcerative colitis (UC) of 4 years of diagnosis. She debuted with acute severe ulcerative colitis in 2017 , with the need of IV steroids with poor response, therefore needing induction with Infliximab with adequate response. However, at the end of 2019 she required optimization due to low infliximab levels, with no antibodies with good response. In October of 2020 therapeutic monitoring showed low levels again and no antibodies, thus requiring change of therapeutic target to vedolizumab with early clinical response at 6 weeks. Currently she remains with clinical remission and negative fecal calprotectin levels.
\end{abstract}

Key words: Ulcerative colitis. vedolizumab. Second line therapy.

\section{Presentación de caso clínico}

Mujer de 24 años con diagnóstico de colitis ulcerosa (CU) en mayo de 2017, con inicio de síntomas en enero del mismo año, tratada inicialmente por sospecha de parasitosis con múltiples esquemas antibióticos y antiparasitarios. En mayo del 2017 acude a urgencias por presentar cuadro de cinco semanas de evolución con incremento en el número de evacuaciones en comparación con el basal de 5 al día hasta 15 al día, con incremento en cantidad de sangre, moco y tenesmo, con incontinencia ocasional; acompañadas con dolor abdominal con predominio en hipogastrio, fiebre hasta de $39^{\circ} \mathrm{C}$ en la última semana y pérdida de peso de 7 kilos en los últimos 
dos meses. Las pruebas de laboratorio a su ingreso con datos de anemia grave, con hemoglobina $(\mathrm{Hb})$ $4.5 \mathrm{~g} / \mathrm{dl}$, hematocrito del $14.2 \%$, leucocitosis de 13.400 células $/ \mathrm{mcl}$, neutrófilos 10.600 células $/ \mathrm{mcl}$, trombocitosis de 854.000 células/mcl, hipoalbuminemia con $2.1 \mathrm{~g} / \mathrm{dl}$, con elevación de proteína $\mathrm{C}$ reactiva hasta $86.4 \mathrm{mg} / \mathrm{l}$. Debido a sus condiciones generales se decide su hospitalización en la unidad de cuidados intermedios, colocación de catéter central y se realiza transfusión de cuatro concentrados eritrocitarios; se inició tromboprofilaxis con enoxaparina $40 \mathrm{mg}$ subcutáneos cada $24 \mathrm{~h}$ y apoyo con nutrición parenteral total calculada por nutrición clínica. Debido a los datos de sangrado de tubo digestivo bajo, se realizó colonoscopia una vez estabilizada la paciente en las primeras $24 \mathrm{~h}$ de su ingreso. Se realizó colonoscopia corta hasta ángulo esplénico por riesgo de perforación, con hallazgo de colitis inflamatoria extensa (más allá del ángulo esplénico), con morfología tubular del colon, con sangrado espontáneo, friabilidad de la mucosa y pérdida completa del patrón vascular de la mucosa. Se tomaron biopsias de descendente, sigmoides y recto, con reporte histopatológico de cambios compatibles con CU crónica idiopática con actividad histológica grave por índice de Robarts, sin cambios citopáticos compatibles con citomegalovirus. Como parte del abordaje se solicitó a su ingreso exámenes en heces siendo el panel de reacción en cadena de la polimerasa (PCR) gastrointestinal (Biofire-FilmArray) negativo para bacterias, virus y parásitos. Con los hallazgos comentados previamente, se decide inicio de hidrocortisona $100 \mathrm{mg}$ cada 6 h, y mesalazina 1.5 gramos vía oral cada 8 h, con mejoría parcial de los síntomas y número de evacuaciones; sin embargo, durante la evaluación al tercer día de tratamiento con esteroides intravenosos (IV) por criterios de Oxford la paciente persistía con 9 evacuaciones al día y proteína $C$ reactiva de $67.5 \mathrm{mg} / \mathrm{l}$, por lo que se decide iniciar manejo con infliximab. Cabe destacar que en nuestra institución se realiza protocolo de enfermedades infecciosas y valoración por un equipo multidisciplinario de infectología, coloproctología, hematología, nutrición clínica y las especialidades afines de acuerdo con las necesidades del paciente desde el momento de ingreso o del diagnóstico de la $\mathrm{CU}$, por lo que para el $4 .^{\circ}$ día de ingreso de la paciente ya se contaba con pruebas negativas para tuberculosis, virus de inmunodeficiencia humana y hepatitis (la paciente contaba con esquema completo para hepatitis $B$ y con inmunización adecuada), por lo que se decidió en conjunto con la paciente el inicio de infliximab como terapia de rescate a dosis de $5 \mathrm{mg} / \mathrm{kg}$, por lo que correspondió una dosis de $260 \mathrm{mg}$ IV de infliximab; a las $48 \mathrm{~h}$ de la aplicación de infliximab la paciente se encontraba con 4 evacuaciones al día, sin sangre, sin dolor abdominal, sin fiebre y con proteína $C$ reactiva de $22 \mathrm{mg} / \mathrm{l}$. Se decidió su egreso al décimo día de hospitalización con $\mathrm{Hb}$ de $10 \mathrm{mg} / \mathrm{dl}$, albúmina en $2.7 \mathrm{~g} / \mathrm{dl}$ y en mejores condiciones generales, con esquema de prednisona vía oral de dosis reducción y se inicia azatioprina a dosis de $50 \mathrm{mg}$, con posterior optimización a $125 \mathrm{mg}$ tres semanas después. La paciente continuó su seguimiento en la consulta de enfermedad inflamatoria, con aplicación de las siguientes dosis de infliximab en la semana 2 y 6 , con mejoría en parámetros bioquímicos y con remisión clínica completa en la semana 10, por lo que se decide realizar nueva colonoscopia en la semana 12 para evaluar nuevamente extensión y respuesta endoscópica al biológico, con hallazgo de remisión endoscópica completa con puntuación 0 en la escala de Mayo endoscópica, y con resolución total de la anemia. Se continúa esquema con infliximab cada ocho semanas con monitorización con niveles séricos con promedio de $3.9 \mathrm{mcg} / \mathrm{ml}$ y anticuerpos previo a cada infusión con cuantificaciones bajas, y vigilancia de calprotectina fecal cada cuatro meses, con mantenimiento de la remisión, con calprotectina fecal promedio de $125 \mathrm{mcg} / \mathrm{g}$ hasta finales de junio del 2019, cuando se solicitan nuevos niveles de calprotectina de $260 \mathrm{mcg} / \mathrm{g}$ y niveles de infliximab tres semanas después de la dosis previa, con niveles de $0.4 \mathrm{mcg} / \mathrm{ml}$ con anticuerpos negativos, por lo que se decide optimizar dosis a cada cuatro semanas y se recalcula por peso recuperado de la paciente a $280 \mathrm{mg}$, la siguiente dosis recupera niveles séricos a $3.5 \mathrm{mcg} / \mathrm{ml}$ y disminuye la calprotectina a $200 \mathrm{mcg} / \mathrm{g}$. La paciente se mantiene en remisión clínica, sin embargo, en nuevo control en octubre del 2020 aunque la calprotectina se mantuvo en $220 \mathrm{mcg} / \mathrm{g}$, los niveles de infliximab bajaron nuevamente a $1.2 \mathrm{mcg} / \mathrm{ml}$, por lo que se decide en conjunto con la paciente modificar blanco terapéutico hacia antiintegrinas debido a pérdida de la respuesta secundaria al infliximab. Previo a la modificación de la terapia se realizan nuevos exámenes de heces y nueva colonoscopia con biopsias para evaluar nuevamente extensión y descartar citomegalovirus sin hallazgos de cambios citopáticos secundarios a esta infección, sin embargo, con actividad endoscópica Mayo 3 (Fig. 1) y reporte histopatológico de actividad moderada de colitis ulcerosa crónica inespecífica en 
sigmoides y recto (Fig. 2). Por lo que se realiza infusión de vedolizumab $300 \mathrm{mg}$ IV en las semanas 0, 2 y 6 , con seguimiento de la calprotectina fecal a las seis semanas de la última dosis de inducción con niveles de $78 \mathrm{mcg} / \mathrm{g}$. Actualmente la paciente continúa con infusión cada ocho semanas de vedolizumab, con niveles actuales de calprotectina fecal de $60 \mathrm{mcg} / \mathrm{mg}$, con remisión clínica, no ha requerido nuevas hospitalizaciones y se mantiene sin anemia y libre de esteroides.

\section{Discusión y revisión}

La terapia con agentes contra el factor de necrosis tumoral alfa (anti-TNF- $\alpha$ ) como tratamiento de primera línea en la CU ha demostrado un gran avance en la inducción a la remisión y mantenimiento de la enfermedad moderada a severa, reduciendo el riesgo de cirugía, hospitalización y de complicaciones relacionadas con la enfermedad. Sin embargo, existen reportes de que algunos pacientes pueden no responder (falla primaria), un $30-40 \%$, además se puede presentar la pérdida de la respuesta con el tiempo (pérdida de respuesta secundaria) hasta en otro $30-40 \%$; existen reportes de pérdida de respuesta secundaria hasta en un $60 \%$ de pacientes con respuesta inicial a anti-TNF en los primeros cinco años del tratamiento². También pueden existir pacientes intolerantes a la terapia inicial con anti-TNF y hasta el momento no se han identificado qué factores puedan predecir la falla o pérdida de respuesta con anti-TNF. Se puede concluir que aquellos pacientes tratados previamente con algún anti-TNF están mayormente predispuestos a una segunda falla al tratamiento, probablemente debido a una alteración inmunitaria a mayor número de exposición a fármacos biológicos. Por este motivo se han estudiado otros agentes con diferentes blancos moleculares para el tratamiento de segunda línea en CU, siendo el más estudiado la terapia de antiintegrinas como el vedolizumab.

El vedolizumab es un anticuerpo monoclonal humanizado IgG1 que inhibe selectivamente a la integrina $\alpha 4 \beta 7$ de los linfocitos T. La función de esta integrina es la unión con las moléculas de adhesión del epitelio intestinal, por lo tanto su inhibición resulta en una menor respuesta inflamatoria al disminuir la migración de linfocitos activados hacia el área de inflamación ${ }^{3}$. Dentro de las ventajas del vedolizumab, al ser selectivo de esta integrina intestinal, está que se pueden evitar las complicaciones sistémicas y neurológicas

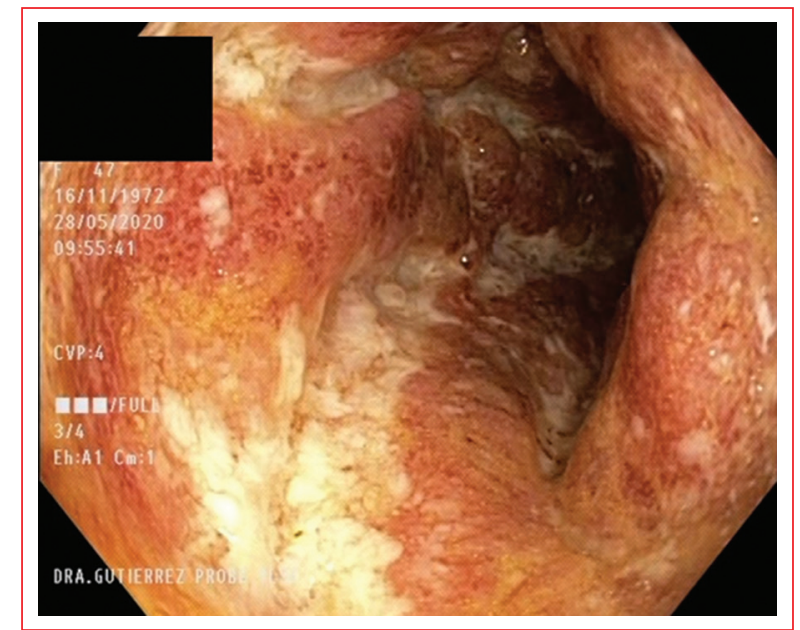

Figura 1. Colonoscopia. Imagen de sigmoides con actividad endoscópica Mayo 3.

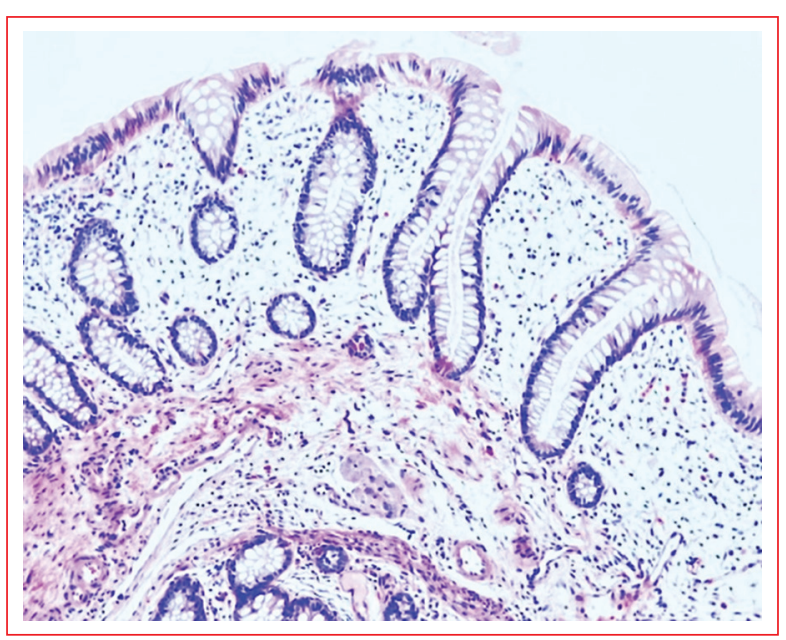

Figura 2. Imagen histológica. Cambios en la estructura de las criptas: criptitis, abscesos crípticos y ramificación. No se observaron datos de infección por CMV.

observadas con otros fármacos antiintegrinas, como el natalizumab ${ }^{4}$.

Desde la aprobación del vedolizumab en el 2014 se ha demostrado su efectividad y seguridad en la inducción a la remisión y mantenimiento como tratamiento de primera línea en pacientes con $\mathrm{CU}$, con un buen perfil de seguridad que incluye menor riesgo de infecciones en comparación de la terapia con anti-TNF ${ }^{5}$. Así mismo, los estudios pivotales evaluando la terapia con vedolizumab han demostrado un buen resultado en pacientes tratados previamente con terapia anti-TNF. 
El estudio del consorcio VICTORY, el cual tuvo como objetivo evaluar el uso de vedolizumab en CU en desenlaces de remisión clínica y endoscópica a 6 y 12 meses junto con otros desenlaces secundarios, demostró que la exposición previa a un anti-TNF se asoció con una menor probabilidad de respuesta clínica (hazard ratio [HR]: 0.71), remisión clínica (HR: 0.52), mejoría endoscópica y remisión endoscópica (HR: 0.51), en comparación con los pacientes vírgenes a terapia biológica ${ }^{6}$. En un reporte post hoc del estudio GEMINI 1, que comparó vedolizumab vs. placebo, en aquellos pacientes con falla al tratamiento con anti-TNF se observó una diferencia del 36 contra el 5.3\% en la remisión clínica a 52 semanas, favoreciendo la terapia con vedolizumab como segunda línea y con una mejor respuesta en aquellos con intolerancia a anti-TNF que los que presentaron falla a la respuesta primaria con estos ${ }^{7}$. En el estudio VARSITY, que comparó vedolizumab frente a adalimumab, el subgrupo de pacientes que habían recibido un anti-TNF previo demostró una mayor tendencia a favor del vedolizumab en la remisión clínica y endoscópica, no así en la remisión libre de esteroide ${ }^{8}$.

En años recientes se ha investigado el uso del vedolizumab como terapia de segunda línea en pacientes con falla previa a los anti-TNF, con pocos estudios de la vida real diseñados con este objetivo. En un estudio italiano se observó una menor tasa de falla al tratamiento con el uso de vedolizumab (49\%) en comparación con adalimumab (26\%), en pacientes con CU tratados previamente con infliximab ${ }^{9}$. En otro estudio observacional pacientes con $\mathrm{CU}$ expuestos a un antiTNF subcutáneo (adalimumab o golimumab) se evaluó la efectividad y seguridad del vedolizumab como terapia de segunda línea comparado con infliximab. En la semana 14 , un $26 \%$ del grupo de infliximab alcanzaron la remisión clínica comparado con un $49 \%$ del grupo de vedolizumab, demostrando ser dos veces superior la terapia con vedolizumab, no solo para la remisión clínica, sino también en tiempo libre de esteroide, cirugía y eventos adversos ${ }^{10}$. Finalmente, en un estudio reciente en Suecia, en pacientes con $\mathrm{CU}$ expuestos previamente a anti-TNF, se comparó la tasa de respuesta de vedolizumab frente a otro agente anti-TNF. Se observó una mayor supervivencia a 12 meses del $69 \%$ en el grupo de vedolizumab comparado con el $62 \%$ en el grupo anti-TNF; con una diferencia porcentual de 7 puntos. No se observaron diferencias en el perfil de seguridad, hospitalizaciones ni infecciones entre ambos grupos, concluyendo que ambas terapias son comparables en su perfil de seguridad y efectividad ${ }^{11}$.

Por lo tanto, la evidencia se demuestra a favor del vedolizumab como terapia de segunda línea en pacientes con CU tratados previamente con un anti-TNF, si bien aún no existen estudios prospectivos para concluir la superioridad del vedolizumab. Con el aumento en la incidencia de enfermedad inflamatoria intestinal y la mayor disponibilidad de nuevas terapias biológicas y otros blancos moleculares, es importante determinar la probabilidad de respuesta a agentes de segunda línea en pacientes expuestos previamente a una terapia con anti-TNF para lograr así el desarrollo de algoritmos terapéuticos en estos pacientes. En el presente caso de la vida real, mostramos la respuesta favorable del vedolizumab como tratamiento de segunda línea para el manejo de $\mathrm{CU}$ en un paciente expuesto previamente a un anti-TNF.

\section{Financiamiento}

Los autores declaran no haber recibido financiación para este estudio.

\section{Conflicto de intereses}

Los autores declaran no tener conflicto de intereses.

\section{Responsabilidades éticas}

Protección de personas y animales. Los autores declaran que para esta investigación no se han realizado experimentos en seres humanos ni en animales.

Confidencialidad de los datos. Los autores declaran que han seguido los protocolos de su centro de trabajo sobre la publicación de datos de pacientes.

Derecho a la privacidad y consentimiento informado. Los autores han obtenido el consentimiento informado de los pacientes y/o sujetos referidos en el artículo. Este documento obra en poder del autor de correspondencia.

\section{Bibliografía}

1. Singh S, George J, Boland BS, Vande Casteele N, Sandborn WJ. Primary non-response to tumor necrosis factor antagonists is associated with inferior response to second-line biologics in patients with Inflammatory bowel diseases: A systematic review and meta-analysis. J Crohns Colitis. 2018;12(6):635-43.

2. Ma C, Huang V, Fedorak DK, Kroeker KI, Dieleman LA, Halloran BP, et al. Outpatient ulcerative colitis primary anti-TNF responders receiving adalimumab or infliximab maintenance therapy have similar rates of secondary loss of response. J Clin Gastroenterol. 2015;49(8):675-82. 
Rev Mex Enferm Inflam Inmunomed. 2021;95(2)

3. Takatsu N, Hisabe T, Higashi D, Ueki T, Matsui T. Vedolizumab in the treatment of ulcerative colitis: An evidence-based review of safety, efficacy, and place of therapy. Core Evid. 2020;15:7-20.

4. Pagnini C, Pizarro TT, Cominelli F. Novel pharmacological therapy in inflammatory bowel diseases: Beyond anti-tumor necrosis factor. Front Pharmacol. 2019;10:671.

5. Bonovas S, Fiorino G, Allocca M, Lytras T, Nikolopoulos GK, Peyrin-Biroulet $L$, et al. Biologic therapies and risk of infection and malignancy in patients with inflammatory bowel disease: A systematic review and network meta-analysis. Clin Gastroenterol Hepatol. 2016;14(10):1385-97.

6. Narula N, Peerani F, Meserve J, Kochhar G, Chaudrey K, Hartke J, et al. Vedolizumab for ulcerative colitis: Treatment outcomes from the VICTORY Consortium. Am J Gastroenterol. 2018;113(9):1345-54.

7. Feagan BG, Rubin DT, Danese S, Vermeire S, Abhyankar B, Sankoh S, et al. Efficacy of vedolizumab induction and maintenance therapy in patients with ulcerative colitis, regardless of prior exposure to tumor necrosis factor antagonists. Clin Gastroenterol Hepatol. 2017;15(2):229-39.e5.
8. Sands BE, Peyrin-Biroulet L, Loftus EV, Danese S, Colombel J-F, Törüner $M$, et al. Vedolizumab versus adalimumab for moderate-to-severe ulcerative colitis. N Engl J Med. 2019;381(13):1215-26.

9. Favale A, Onali S, Caprioli F, Pugliese D, Armuzzi A, Macaluso FS, et al. Comparative efficacy of vedolizumab and adalimumab in ulcerative colitis patients previously treated with infliximab. Inflamm Bowel Dis. 2019:25(11):1805-12

10. Hupé $M$, Rivière $P$, Nancey $S$, Roblin X, Altwegg R, Filippi J, et al. Comparative efficacy and safety of vedolizumab and infliximab in ulcerative colitis after failure of a first subcutaneous anti-TNF agent: a multicentre cohort study. Aliment Pharmacol Ther. 2020;51(9): 852-60.

11. Rundquist S, Sachs MC, Eriksson C, Olén O, Montgomery S, Halfvarson J. Drug survival of anti-TNF agents compared with vedolizumab as a second-line biological treatment in inflammatory bowel disease: results from nationwide Swedish registers. Aliment Pharmacol Ther. 2021; $53(4): 471-83$ 